\title{
Caracterización de Personas que Conviven con VIH en la Ciudad de Cartagena (Bol. - Col)
}

\section{Characterization of People Who Live with HIV in Cartagena}

\author{
Recibido: 10 de Oct. 2013/Enviado para modificación: 30 de Nov. 2013/Aceptado: 14 de En. 2014
}

Irma Yolanda Castillo Ávila ${ }^{1}$

Universidad de Cartagena

\section{RESUMEN}

Introducción: El Virus de Inmunodeficiencia Humana (VIH) constituye uno de los desafíos para la salud pública, por ser una patología de incremento constante. Objetivo: Describir las principales características de las personas que conviven con el VIH en la ciudad de Cartagena. Materiales y Métodos: Se realizó un estudio descriptivo, durante tres meses. La población objeto de estudio fue conformada por 763 personas con el diagnostico. Se estimó un tamaño de muestra de 219 personas, que fueron incluidas mediante un muestreo no probabilístico. Como instrumento de recolección de información, previo consentimiento informado, se utilizó una encuesta con tres apartados: Apartado 1: Características demográficas, Apartado 2: características socio-familiares, y Apartado 3: características socio-sanitarias. Para el análisis de los datos, se aplicó la estadística descriptiva; se calcularon medias, medianas y desviaciones estándar. Resultados: Un total de 201 personas diligenciaron el cuestionario satisfactoriamente. El 62\% (124) de estas fueron de sexo masculino, y la media de edad, de 38 años (DE: 11); un 59,7\% (120) procede del área rural; 49\% (98) cuenta con bachillerato completo o incompleto. Además, el 66\% (132) convive con 3 a 6 personas, 45\% (91) tiene parejas estables, 84\%(169) son de estratos 1 y 2; en tanto que el 85,6\% (172) manifestó no tener vinculo laboral con una empresa. Por último, el 41\% (82) está afiliado al régimen subsidiado y el 99,5\% (200) reportó la vía sexual como posible modo de transmisión. Conclusión: La infección se presenta con mayor frecuencia en hombres de estratos bajos, quienes manifiestan como modo de transmisión la vía sexual. Esto supone retos para los programas de salud sexual y reproductiva

Palabras Clave: Características de la población, infecciones por VIH, empleo, vivienda, seguridad social (Fuente: DeCS).

\section{ABSTRACT}

Introduction: The Virus of Human Immunodeficiency (HIV) constitutes one of the challenges for public health, for being pathology of constant increase. Objective: To describe the main characteristics of people who live with HIV in Cartagena. Materials and Methods: A descriptive study with 763 people with HIV diagnosis over a three months period was conducted. 219 people were sampled as non-probability sample. A three sections consentual was applied as a tool for collecting the relevant information: section 1: demographic characteristics, section 2: social-family features, and paragraph 3: socio-sanitary characteristics. For data analysis, descriptive statistics were applied; means, medians and standard deviations were calculated Results: 201 people answered the questionnaire satisfactorily. The 62\% (124) of these were male, with an average age of 38 years (from: 11); a 59.7\% (120) comes from rural area; 49\% (98) have completed or incomplete secondary education. In addition, 66\% (132) coexists with 3 to 6 people, $45 \%$ (91) has stable couples, $84 \%$ (169) are from strata 1 and 2; as to the $85.6 \%$ (172) reported not having labor ties with a company. Finally, $41 \%$ (82) is affiliated to the subsidized regime and $99.5 \%$ (200) reported the sexually as a possible mode of transmission. Conclusion: Infection occurs in men of lower strata who were more frequently infected by sexual transmission.

Keywords: Population Characteristics, HIV Infections, Employment, Housing, Social Security (Source: MeSH, NLM).

Para citar este artículo: Castillo AI. Caracterización de personas que conviven con VIH en la ciudad de Cartagena. Cienc. innov. salud. 2014; 2 (1):4-10.

1 Enfermera. Especialista en Gerencia en Salud. Magister en Salud Pública. Universidad de Cartagena. Correo electrónico: icastilloa@unicartagena.edu.co 


\section{Introducción}

El Virus de Inmunodeficiencia Humana (VIH) constituye uno de los desafíos más importantes en enfermedades infecciosas para la salud pública, por ser una patología de incremento constante (1). A finales de 2010, aproximadamente 34 millones de personas vivían con el VIH en todo el mundo. Se estimaba que 30 millones de estas eran adultas, y 3.4 millones, menores de 15 años. África, una región que representa solo el $12 \%$ de la población mundial, sigue siendo la más afectada por este virus, pues, alrededor del $68 \%$ de todas las personas que vivían con el VIH residían en este país $(1,2)$.

En América Latina, se calcula que hay tres millones de afectados. Y desde 1996, las estadísticas presentan un descenso constante en cuanto a las nuevas infecciones por el virus; estabilizándose en los primeros años del nuevo milenio en 100.000 casos por año $(2,3)$. Según el informe mundial de avances de lucha contra el VIH, desde 1985 al 31 de diciembre de 2011, se han reportado en Colombia un total de 75.620 casos de infección por VIH (2), y cada año se diagnostican 4200 casos nuevos $(3,4)$. Más específicamente, en el departamento de Bolívar, la tasa de mortalidad asociada a esta enfermedad muestra una tendencia hacia el aumento. Así, en 2009, dicho departamento registró una prevalencia de 6.4 casos por cada 100 mil habitantes, 1.5 más que en $2001(4,5)$. En la capital del departamento, Cartagena, se diagnosticaron 267 casos en el año 2010, de los cuales el 22,5\% había desarrollado el SIDA, y $12,4 \%$ terminaron con el deceso del paciente (4-7).

El rápido avance de la epidemia en Latinoamericana pone de manifiesto la necesidad de realizar acciones urgentes y eficaces. En la actualidad, la enfermedad es considerada una prioridad de salud pública y las actividades preventivas se han incluido en las metas de la OMS para el año 2015 (8). Pero, para lograr esto, se requieren cambios estructurales en los sistemas de seguridad social, que permitan mejores mecanismos de prevención y un mejor y mayor impacto de los programas (4-6).

De acuerdo con la situación anterior, en Colombia se han implementado medidas para aumentar la afiliación de los pacientes afectados al Sistema General de Seguridad Social en Salud, a fin de lograr una mejor cobertura, atención sanitaria y provisión de terapia a todos los pacientes identificados que la requieran. No obstante, en la actualidad, del total de personas que requieren medicamentos, solo el $71 \%$ lo recibe y aun persisten dificultades para el acceso al diagnóstico, la identificación oportuna de los casos, y el inicio temprano de la terapia. Todo ello limita la reducción de los costos que genera la incapacidad y muerte por las complicaciones derivadas de la enfermedad $(4,5$, 9).

Para el año 2012, la población más afectada por la enfermedad en Colombia, se ubicaba en el rango de los 20 a 39 años (52.7\%), observándose un aumento paulatino del número de casos reportados en los últimos años. Es interesante al respecto que el aumento de los casos fue más marcado entre los mayores de 60 años. Asimismo, la prevalencia de casos en mujeres gestantes es menor al 1\%, mientras que en los hombres que tienen relaciones sexuales con otros hombres es del 5\% (8-10).

La forma más común de trasmisión de la enfermedad sigue siendo la sexual, mostrando una tendencia de avance rápido. En este sentido, resulta preocupante que la mayoría de la población en riesgo y que convive con el virus hace poco uso del condón. Como principales razones para no usarlo, las personas aducen que perciben una disminución de placer, que sienten vergüenza de hacerle esa petición a la pareja y que lo olvidan cuando se encuentran bajo el efecto de alguna sustancia psicoactiva. En este orden de ideas, algunas investigaciones señalan que la población joven puede tener conocimientos sobre la enfermedad, pero no son coherentes con la sensación de riesgo y con la adopción de prácticas que los pueden proteger $(6,10)$.

Para quienes ya tienen el diagnostico, el sistema debe garantizar la estructuración de políticas y servicios de promoción de la salud de manera integral, con énfasis en la salud sexual y reproductiva, para evitar el desgaste y las muertes prematuras, debido a la mayor vulnerabilidad de esta población $(6,9,10)$.

A pesar de los esfuerzos realizados hasta el momento para lograr el aseguramiento universal y mejorar la asistencia en salud en la población, persisten deficiencias programáticas y financieras, que dificultan la tarea preventiva en VIH y SIDA. Particularmente, el acceso universal a asistencia y terapia antirretroviral, solo será posible una vez se alcance la universalización en coberturas de aseguramiento en salud, ye acceso universal a prevención requiere un fuerte compromiso por parte de 
los responsables de las acciones de salud colectivas y de otros sectores, principalmente al interior de las poblaciones más afectadas o en mayores condiciones de vulnerabilidad $(7,9,10)$. Por todo lo anterior, en este estudio se propuso estudiar las características generales de las personas con VIH.

\section{Materiales y Métodos}

Se realizó un estudio descriptivo, durante tres meses. La población objeto de estudio estuvo constituida por 763 personas diagnosticadas con $\mathrm{VIH}$, de acuerdo con el reporte del Departamento Administrativo Distrital de Salud (DADIS) de la ciudad de Cartagena. Se estimó un tamaño de muestra de 219 personas con un nivel de significancia del $95 \%$, un error de $5 \%$, una proporción de infección del $18 \%$ y un porcentaje de pérdidas del $20 \%$.

Se aplicaron encuestas a personas diagnosticadas mayores de edad, que autorizaron su participación y que asistían a citas de control en los centros de atención ambulatorios y hospitalarios de la cuidad que atienden estos pacientes. Las encuestas fueron aplicadas, previo entrenamiento, por las enfermeras coordinadoras encargadas de la consulta de control, en dos centros especializados a la que asistían los pacientes. Esto garantizó la confidencialidad del diagnostico.

Para la recolección de información, se aplicaron los principales ítems del cuestionario SIVIGILA, que mide las variables demográficas. De la historia clínica, se extrajo el perfil clínico e inmunológico, incluyendo el estadio de la clasificación CDC, carga viral, linfocitos TCD4, tiempo de infección por el VIH en meses y el posible modo de trasmisión. En cuanto a las variables económicas, se aplicó un cuestionario, diseñado por los investigadores, en el que se registraron las principales actividades económicas y laborales de los participantes del estudio.

Para el análisis estadístico, se usó el programa SPSS, y el comportamiento de los datos y la probabilidad de error aleatorio se analizaron mediante estadística descriptiva. En este sentido, se calcularon medias, medianas y desviaciones estándar como estimadores descriptivos del comportamiento de los datos.

Teniendo en cuenta los criterios éticos estipulados en la Resolución 08430 del 4 de Octubre 1993 del Ministerio de Salud Publica, se solicitó consentimiento informado por escrito, exponiendo los objetivos del estudio, que aplican para una investigación descriptiva. Además, se atendieron las normas para la investigación en personas que conviven con VIH (11).

\section{Resultados}

En definitiva, participaron del estudio 201 personas con diagnostico de VIH. La distribución de los encuestados con relación al sexo fue de 62\% (124) masculino, (Figura 1), y la media de edad, de 38 años (DE: 11). El 50\% de la población era menor de 39 años, y el mínimo de edad fue de 17 años.

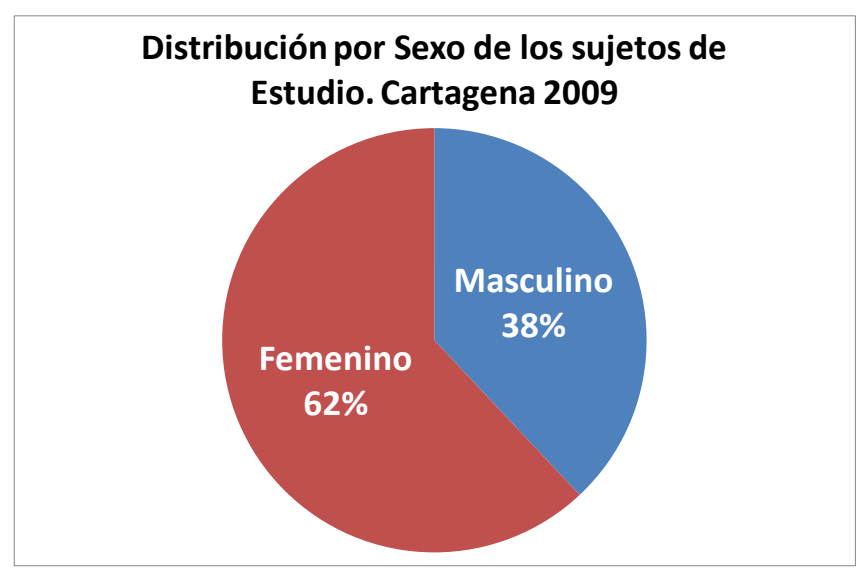

Figura 1. Sexo de los sujetos de estudio

\section{Características demográficas de los sujetos}

Las principales características demográficas de los participantes se relacionan con la procedencia. Así, el $40.3 \%$ (81) era de origen urbano, mientras que un $59.7 \%$ (120), era rural. El $12.4 \%$ (25) procedía de zonas distintas al distrito o departamento. El $45.3 \%$ (91) de los participantes manifestó tener una relación de pareja estable, bien sea por ser casados o vivir en unión libre. En cuanto a la escolaridad, se observó un nivel de escolaridad medio, en su mayoría bachillerato incompleto o completo (49\% (98)); sin embargo, una proporción considerable (19\%, 41 entrevistados) posee un nivel de formación técnico o superior.

El 100\% (201) manifestó pertenecer o tener afinidad, por algún tipo de grupo religioso, mayoritariamente por la religión católica (83\% (166)), seguido de los que dicen ser "cristianos" o "evangélicos" (15\% (30)). 
Del total de pacientes con el VIH, 66\% (132) convive con 3 a 6 personas. En promedio, estos pacientes tienen 4 hijos, dentro y fuera de la unión. En su mayoría, habitan en viviendas familiares $(55 \%$ (111)), que poseen adecuadas características de estructura. La distribución de los servicios públicos de estas viviendas es así: cuentan con servicio de agua, el 93\% (187); alcantarillado, 69\% (139); luz eléctrica, 99\% (198); gas natural, 74\% (149); y teléfono, 51\% (102).

La distribución por estratos de los participantes fue bastante homogénea, encontrándose el mayor porcentaje en los estratos 1 y 2 (84\%(169)), el numero más bajo de participantes pertenecía al estrato $5(0,5 \%$ (1)).

\section{Características económicas}

Del total de personas entrevistadas, el $86 \%$ (172) manifestó no tener ningún tipo de vinculo laboral en la actualidad (Figura 2). Al indagar, además, por la experiencia laboral, se encontró que en promedio tienen 20 meses de experiencia laboral, siendo el mayor tiempo de experiencia 360 meses, y el menor de un mes. Es interesante notar que el promedio de permanencia en el trabajo actual es de 9 meses, lo que da un indicio de la estabilidad laboral de estas personas en términos de duración y permanencia laboral.

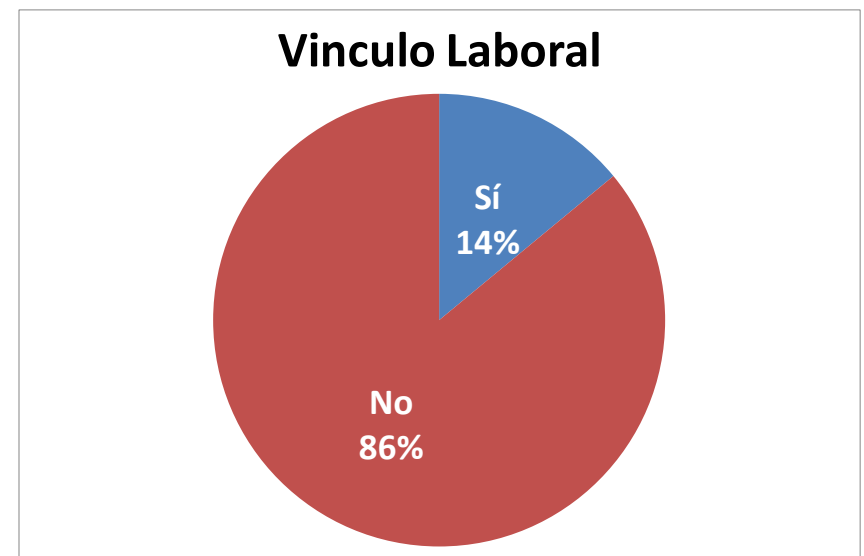

Figura 2. Vinculo laboral de los participantes del estudio

Del total de personas que manifestaron tener vínculo laboral con una empresa (14\% (29)), 3 (10\%) pertenecían a empresas del sector público, y 26 (90\%), a empresas del sector privado.

Con relación al tipo de contratación, $41 \%$ (12) afirmó tener contratos a término fijo, 34\% (10), a termino indefinido, y $14 \%$ (4), por prestación de servicios. De todos estos, 79\% (23) está afiliado al sistema de seguridad social; $72 \%$ (21), a un fondo de pensiones; $72 \%$ (21), a un fondo de cesantías; y 79\% (23), a una aseguradora de riesgos profesionales. El $86 \%$ (25) dijo no estar agremiado y el 90\% (26) indicó que no pertenecía al sindicato de la empresa donde laboraban.

Las horas laboradas al día por estas personas osciló entre 6 y 10, con un promedio general de 8. Las horas laboradas por semana iban de 70 a 20, con un promedio de 47. Además, quienes manifestaron tener vínculo laboral, en promedio contaban un (1) día de descanso por semana.

Con relación a los ingresos, estos oscilaron entre 50.000 y 2.500 .000 pesos mensuales, con un promedio de 394.204 pesos. Al respecto, la mediana y la moda fueron iguales para este grupo: 300.000 pesos. Con relación a la percepción de ingresos de fuentes distintas de una relación laboral, se encontró que para el 27\% (55) sus ingresos dependen de su pareja, $4 \%$ (9) son pensionados, y el 38\% (77) realiza su actividad económica en casa o trabaja como comerciante. Al indagar sobre sus oficios, el 15\% (30) se definió como "amas de casa", 7\% (14), como "vendedor", y 3\% (6), como "estudiantes".

\section{Características clínicas}

El tiempo de haber sido diagnosticado osciló entre 21 años y un (1) año. Concretamente, el 56\% (113) tiene menos de 4 años de diagnóstico (diagnosticados desde el año 2004 al 2008), en tanto que el 24\% (49) fue diagnosticado entre los años 1999 y 2002. Y para el 100\% (201) de estos diagnósticos, se utilizo como prueba de laboratorio confirmatoria el Western-Blot.

En el 99.5\% (200) de los casos, se reportó como posible modo de transmisión la vía sexual, y el $0.5 \%$ (1) restante reportó la transmisión vertical.

Del total de participantes, al 54,7\% (110) se le había realizado, en los últimos seis (6) meses antes de la 
encuesta, pruebas de laboratorio para determinar la carga viral y el recuento linfocitario.

El 97.5\% afirmó que recibe tratamiento suministrado de su aseguradora (contributiva o subsidiada), y el $2.5 \%$ (5) restante manifestó no recibirlo. Con relación a la asistencia a consulta en el mes anterior, el 83\% (167) expresó haberlo hecho, el 13,4\% (27) había consultado hacía dos meses, y el 3,6\% (7) restante manifestó haber realizado la consulta hacia más de dos meses

\section{Discusión}

Los trastornos alimenticios pertenecen a la clasificación de enfermedades psicosomáticas constituyen uno de los principales problemas de salud más frecuentes en los adolescentes, asociados incluso a otros tipos de patologías, como demencia y suicidio. El porcentaje de este último se encuentra entre el 1.8 al $7.3 \%$ asociado con algún tipo de TCA $(19,20)$.

La bulimia y la anorexia nerviosa son los trastornos más frecuentes a nivel psiquiátrico. Lo anterior se demostró con el tamizaje realizado en la presente investigación, al identificar las características psicológicas y comportamentales como enfermedades psicosomáticas que figuran entre los problemas de salud crónicos más frecuentes en los adolescentes, sobre todo entre edades de 10 a 24 como lo señalan otros autores $(2,21)$, que nuestro estudio corrobora entre edades de 16 a 20.

La detección temprana o tamizaje podría disminuir la letalidad de estos trastornos, que se asocian con el suicidio o con otros tipos de trastornos psiquiátricos, aunque la anorexia y bulimia nerviosa son las más detectadas. La presente investigación indicó, en el cuadro de características psicológicas y comportamentales, que la imagen corporal y la baja autoestima por la influencia sociocultural median de alguna manera el comportamiento psicológico de los adolescentes y jóvenes (3). En conjunto, estos rasgos predisponen de manera individual a cada estudiante a un tipo de trastorno psicosomático específico que se relaciona con los trastornos alimenticios (22).

Dados los resultados descritos, entre las principales recomendaciones de nuestro estudio aparece la necesidad de implementar estudios longitudinales de tipo transcultural, para plantear estrategias de seguimiento con criterios específicos y establecer el diagnóstico del tipo de trastorno alimenticio o psicosomático al cual están predispuestos los estudiantes (23)

Las diferentes subescalas del EDI mostraron los rasgos psicológicos y comportamentales comunes a la anorexia nerviosa y bulimia nerviosa en los estudiantes de los 5 programas. El miedo a madurar fue la variable más alta en todos los análisis realizados, seguida por el perfeccionismo. La investigación sugirió, además, que es necesario identificar los factores de riesgo que influyen en las mujeres para que padezcan este tipo de trastornos de conducta alimentaria, entre los que se cuentan: factores sociales, ambientales y personales como la insatisfacción o la obsesión, los cuales se cuentan entre los rasgos psicológicos mostrados en la Tabla 2.

En el grupo de estudiantes, se percibe un riesgo hacia los trastornos alimentarios como la ingesta y el impulso a la delgadez que aparecen como uno de los síntomas más predisponentes a la bulimia y anorexia nerviosa, debido a los dramáticos esfuerzos por controlar el peso y la silueta, además de los diferentes trastornos condicionados por la falta de autoestima: insatisfacción corporal, el perfeccionismo como elemento de aceptación social, desconfianza interpersonal y la falta de reconocimiento de sus propias emociones y sensaciones (conciencia interoceptiva). Todo ello sumado al miedo a madurar o a adquirir responsabilidades, sobre todo entre las edades de 16 a 20 años, cuando están por culminar la profesión (Tabla 1).

En síntesis, en el presente estudio, se encontró que los estudiantes corren un alto riesgo de presentar la anorexia y la bulimia nerviosa, debido a los rasgos psicológicos y comportamentales encontrados. Esto conlleva a proponer o diseñar un programa de tamizaje e intervención en todas las escuelas y universidades del país. También se sugiere ampliar el estudio a otros programas de diferentes facultades, tomando en cuenta la intensidad horaria de las clases, falta de tiempo libre, exigencia académica a la hora de los exámenes, exigencias propias de la institución, factores familiares, económicos, socio-culturales, lo cual hizo falta determinar en la presente investigación, debido al principal propósito de la misma. 


\section{Agradecimientos}

A los estudiantes de Enfermería Juan Guillermo Barrantes Vera, Marco Antonio Conrado Patiño, Carlos Arturo Díaz Suazman y Andrés Mauricio Molinares Arismendi, por su colaboración en la aplicación del instrumento a los estudiantes de pregrado. También a los estudiantes, padres, docentes, personal y administrativo de cada una de las dependencias de la universidad, que participaron de manera directa o indirecta del presente estudio. A la Corporación Universitaria Rafael Núñez, por permitir la realización del presente estudio en sus instalaciones y brindarnos el apoyo de los docentes al momento de la aplicación de los instrumentos a los estudiantes de la Facultad de Ciencias de la Salud.

\section{Referencias}

1. Cenci M, Peres KG, Vasconcelos FA. Prevalência de comportamento bulímico e fatores associados em universitárias. Rev. psiquiatr. clín. 2009; 36 (3): 83-88.

2. Morandé G, Celada J, Casas JJ. Prevalence of eating disorders in a Spanish school-age population. J. Adolesc. Health. 1999; 24(3): 212219.

3. Harrison K. Body image and eating disorders: definition and prevalence. Routledge Int. Handb. Child. Adolesc. Media. 2013; 224.

4. Trace SE, Baker JH, Peñas-Lledó E, Bulik CM. The Genetics of eating disorders. Annu. Rev. Clin. Psychol. 2013; 9(1): 589-620.

5. Thompson-Brenner H, Franko DL, Thompson DR, Grilo CM, Boisseau CL, Roehrig JP, et al. Race/ethnicity, education, and treatment parameters as moderators and predictors of outcome in binge eating disorder. J Consult Clin Psychol. 2013; 81 (4): 710-721.

6. Gaskin JL, Pulver AJ, Branch K, Kabore A, James T, Zhang J. Perception or reality of body weight: which matters to the depressive symptoms. J. Affect. Disord. 2013; 150(2): 350355.

7. Fandiño A, Giraldo SC, Martínez C, Aux CP, Espinosa R. Factores asociados con los trastornos de la conducta alimentaria en estudiantes universitarios en Cali, Colombia. Rev. Colomb. Médica. 2007; 38(4): 344-351.
8. Alvarenga $\mathrm{M}$ dos $\mathrm{S}$, Lourenço $\mathrm{BH}$, Philippi ST, Scagliusi FB. Disordered eating among Brazilian female college students. Cad. Saúde Pública. 2013; 29(5): 879-888.

9. Fernández ML, Otero MC, Castro YR, Prieto MF. Hábitos alimentarios e imagen corporal en estudiantes universitarios sin trastornos alimentarios. Int. J. Clin. Heal. Psychol. 2003; 3 (1): 23-33.

10. Fairburn CG, Harrison PJ. Eating disorders. The Lancet. 2003; 361(9355): 407-416.

11. Price C, Morgan J. Introducing eating disorders. Medicine (Baltimore). 2012; 14 (1): 97-108.

12. American Psychiatric Association. Diagnostic and statistical manual of mental disorders: DSM-IVTR. Arlington, 2000.

13. Widiger TA, Samuel DB. Diagnostic categories or dimensions? A question for the diagnostic and statistical manual of mental disorders, fifth edition. J. Abnorm. Psychol. 2005; 114 (4): 494504.

14. Yager J. Weighty perspectives: contemporary challenges in obesity and eating disorders. Am. J. Psychiatry. 2000; 157 (6): 851-853.

15. Leal GV da S, Philippi ST, Polacow VO, Cordás TA, Alvarenga $\mathrm{M}$ dos $\mathrm{S}$. What is adolescents' eating disorder risk behavior? J. Bras. Psiquiatr. 2013; 62(1): 62-75.

16. Rodríguez A, Novalbos JP, Martinez JM, Ruiz MA, Fernandez JR, Jimenez D. Eating disorders and altered eating behaviors in adolescents of normal weight in a Spanish city. J. Adolesc. Health. 2001; 28 (4): 338-345.

17. Moya T, Fleitlich-Bilyk B, Goodman R. Brief report: Young people at risk for eating disorders in Southeast Brazil. J. Adolesc. 2006; 29(2): 313317.

18. Pinkston M, Martz D, Domer F, Curtin L, Bazzini $\mathrm{D}$, Smith L, et al. Psychological, nutritional, and energy expenditure differences in college females with anorexia nervosa vs. comparable-mass controls. Eat. Behav. 2001; 2(2): 169-181.

19. Pompili M, Mancinelli I, Girardi P, Ruberto A, Tatarelli R. Suicide in anorexia nervosa: A metaanalysis. Int. J. Eat. Disord. 2004; 36(1): 99-103.

20. Bodell LP, Joiner TE, Keel PK. Comorbidityindependent risk for suicidality increases with bulimia nervosa but not with anorexia nervosa. J. Psychiatr. Res. 2013; 47(5): 617-621. 
21. Hoek HW, Van Hoeken D. Review of the prevalence and incidence of eating disorders. Int. J. Eat. Disord. 2003; 34(4): 383-396.

22. Cruzat C, Ramírez P, Melipillán R, Marzolo P. Trastornos alimentarios $\mathrm{y}$ funcionamiento familiar percibido en una muestra de estudiantes secundarias de la comuna de Concepción, Chile. Psykhe Santiago. 2008; 17(1): 81-90.

23. Brownell KD. Handbook of eating disorders: physiology, psychology, and treatment of obesity, anorexia, and bulimia. Basic Books; 1986. 